Fátima Lopes-da-Silva $\cdot$ Sonia de Pascual-Teresa Julián Rivas-Gonzalo · Celestino Santos-Buelga

\title{
Identification of anthocyanin pigments in strawberry (cv Camarosa) by LC using DAD and ESI-MS detection
}

Received: 5 June 2001 / Revised version: 21 September 2001 / Published online: 7 December 2001

(C) Springer-Verlag 2001

\begin{abstract}
New high performance liquid chromatography (HPLC) conditions were developed for the separation of strawberry anthocyanins that provided a good resolution of peaks at a low flow rate compatible with the requirements of the mass spectrometry (MS) detector. A strawberry extract was fractionated by column chromatography and simple fractions containing basically anthocyanins were obtained, making their analysis by HPLC possible using on-line photodiode array detection and MS. Information on the identity of the major and some secondary anthocyanins in strawberry was obtained from their retention characteristics, UV-visible spectra and mass spectra. The presence in strawberry of the previously reported cyanidin 3-glucoside, pelargonidin 3-glucoside, pelargonidin 3-rutinoside and pelargonidin 3-acetylglucoside was confirmed and cyanidin 3-rutinoside was identified in strawberry for the first time. Furthermore, cyanidin 3-malonyldiglucoside, pelargonidin 3-malylglucoside, a pelargonidin bioside and two possible pelargonidin 3-biosides acylated with acetic acid were also tentatively assigned.
\end{abstract}

Keywords Strawberry · Anthocyanins · Liquid chromatography-mass spectrometry $\cdot$ Cyanidin . Pelargonidin

\section{Introduction}

Despite strawberry (Fragaria x ananassa Duch.) having been the focus of various studies, the anthocyanin composition of this berry still causes some doubts. The most commonly occurring anthocyanins in strawberry are based on cyanidin and pelargonidin (Fig. 1). The main pigment in cultivated strawberries has been identified as

F. Lopes-da-Silva $\cdot$ S. de Pascual-Teresa $\cdot$ J. Rivas-Gonzalo C. Santos-Buelga ( $)$

Laboratorio de Nutrición y Bromatología, Facultad de Farmacia, Universidad de Salamanca, 37007 Salamanca, Spain e-mail: csb@usal.es pelargonidin 3-glucoside and the presence of cyanidin 3 -glucoside is also well documented $[1,2,3,4,5]$.

The use of on-line photodiode array detection (DAD) coupled to high performance liquid chromatography (HPLC) has been shown a useful tool in assisting the characterization of anthocyanins. The UV-visible spectra of the anthocyanins yield information with regard to the nature of the aglycon and the sugar substitution pattern, while the retention characteristics on reversed-phase HPLC are closely related to their polarity and yield information on the nature of the sugar moieties, acylation and substituents of the anthocyanin B-ring $[6,7,8]$. By using this technique, various anthocyanins have been detected in strawberries. Hong and Wrolstad [2] reported the presence of cyanidin 3-glucoside, pelargonidin 3-glucoside, pelargonidin 3-rutinoside (tentative), an unknown pelargonidin derivative and pelargonidin 3-glycoside acylated with acetic acid (tentative), eluted in that order. Similarly, Bridle and García-Viguera [5] identified cyanidin 3-glucoside as the most polar anthocyanin in strawberry, which eluted followed by pelargonidin 3-glucoside (predominant anthocyanin), pelargonidin 3-rutinoside and a less polar pigment proposed to be pelargonidin 3-succinyl-glucoside. Pelargonidin-3-arabinoside was tentatively identified in strawberry by Fiorini [9] based on spectral characteristics and chromatographic

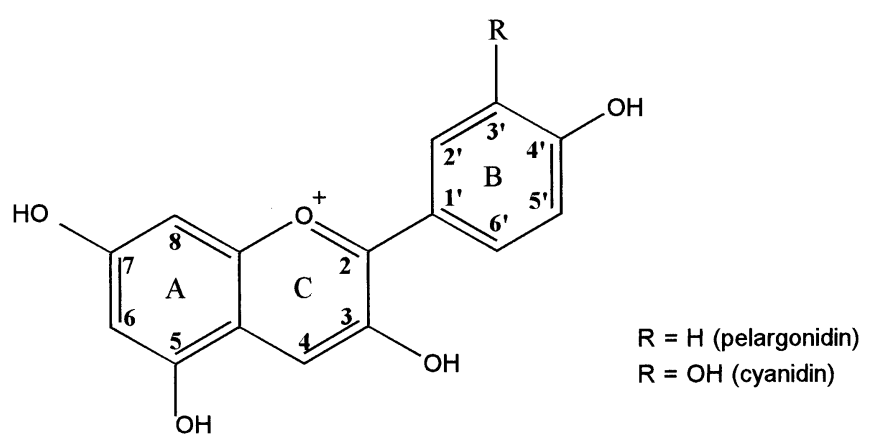

Fig. 1 Basic structure of strawberry anthocyanidins. $R=\mathrm{H}$, pelargonidin; $\mathrm{R}=\mathrm{OH}$, cyanidin 
behaviour. According to Goiffon et al. [10] this would be the third most prevalent anthocyanin (accounting for $3.1-3.9 \%$ of the strawberry pigments) after pelargonidin-3-glucoside $(89-95 \%)$ and cyanidin-3-glucoside $(3.9-10.6 \%)$. Finally, a diglucoside and a trioside of pelargonidin were also suggested to occur in strawberries, as well as a glycoside less hydrophilic than pelargonidin 3 -glucoside, proposed to be a furanose or an anomeric form of pelargonidin 3-glucoside [11].

Nevertheless, the precise identification of most such pigments remains to be solved using appropriate identification techniques other than sequence of elution and absorption spectra. When mass spectrometry (MS) is coupled to a liquid chromatographic separation (LC-MS), both the retention characteristics and the mass spectra provide structural information without requiring the isolation of compounds [12]. If tandem mass spectrometry (MS-MS) techniques are used, it is possible to obtain information concerning the fragmentation pattern of the compounds and consequently, information on their structure and substituents.

This work aimed at developing an HPLC procedure for the separation of strawberry anthocyanins and consequently at establishing and characterizing the anthocyanin composition of strawberries using the absorption and mass spectra obtained from DAD, electrospray ionisation (ESI)-MS and MS-MS detection modes.

\section{Material and methods}

Sample extraction. Strawberries (variety Camarosa), purchased on a local market, were disrupted in $0.1 \% \mathrm{HCl}$ in methanol using a Polytron homogenizer (Kinematica, Littau, Switzerland) and later filtered through a Büchner funnel under vacuum. The solid residue was exhaustively washed with methanol and the filtrates obtained were centrifuged. After addition of water, the supernatant was concentrated under vacuum to total evaporation of the methanol.

Fractionation by column chromatography. The crude strawberry extract was fractionated by column chromatography following a procedure based on that described in Hebrero et al. [7]. A mixture composed of $20 \%$ Polyclar AT and $80 \%$ Kieselgel 60 was boiled under reflux in $\mathrm{HCl}$ for $1 \mathrm{~h}$, and after cooling thoroughly washed with water until a $\mathrm{pH}$ of 5 was reached. The mixture thus prepared was packed up to a height of $30 \mathrm{~cm}$ in a glass column $(50 ¥ 2.5 \mathrm{~cm}$ i.d.) with a filter plate and activated by passing a $\mathrm{MeOH}: 0.1 \%$ $\mathrm{HCl}(20: 80, v / v)$ solution. The aqueous strawberry extract was then carefully deposited onto the stationary phase and washed with water to remove sugars and acids. Further fractionation was performed by elution with EtOH:H2O mixtures containing $0.1 \%$ of $1 \mathrm{~N} \mathrm{HCl}$, establishing a gradient from $20 \%$ to $95 \% \mathrm{EtOH}$. Three fractions (named A, B and C) were collected according to the anthocyanin composition monitored by HPTLC on Kieselgel 60 (Merck), using amyl alcohol:acetic acid:water $(4: 15: 1, \mathrm{v} / \mathrm{v})$ as mobile phase, and these were concentrated under vacuum and recovered in water.

HPLC analysis. The strawberry extract and the anthocyanins fractions were analysed using a Hewlett-Packard 1050 Series liquid chromatograph, equipped with an AQUA (Phenomenex, Torrance, Calif., USA) reversed-phase column (150¥4.60 mm, $5 \mu \mathrm{m}, \mathrm{C} 18)$. The column temperature was thermostatted at $35^{\circ} \mathrm{C}$ using a column heater module (Waters, USA). Solvents were (A) aqueous $0.1 \%$ trifluoracetic acid (TFA) and (B) $100 \%$ HPLC-grade aceto- nitrile, establishing the following gradient: isocratic 10\% B for $5 \mathrm{~min}$, from 10 to $15 \%$ B over $15 \mathrm{~min}$, isocratic $15 \%$ B for $5 \mathrm{~min}$, from 15 to $18 \%$ B over $5 \mathrm{~min}$, and from 18 to $35 \%$ B over $20 \mathrm{~min}$, at a flow rate of $0.5 \mathrm{ml} \mathrm{min-1.} \mathrm{Detection} \mathrm{was} \mathrm{carried} \mathrm{out} \mathrm{in} \mathrm{a} \mathrm{pho-}$ todiode spectrophotometer (SPD M10A VP, Shimadzu, Japan) and $520 \mathrm{~nm}$ selected as preferred wavelength. HPLC solvents and samples were filtered through a $0.45 \mu \mathrm{m}$ Millipore filter (Millipore, Bedford, Mass., USA). Prior to injection of the strawberry extract, a pre-purification was carried out in a C18 Sep-Pak cartridge (Waters), previously activated with methanol followed by $0.1 \mathrm{~N} \mathrm{HCl}$ aqueous solution. The extract adsorbed onto the cartridge was rinsed with ultra-pure water to remove water-soluble compounds like acids and sugars and the pigments were then eluted with MeOH:0.1\% TFA (95:5) solution.

LC/MS analysis. MS was performed using a Finnigan LCQ (Thermoquest, San Jose, USA) equipped with an API source, using an ESI interface. Both the auxiliary and the sheath gas were a mixture of nitrogen and helium. The capillary voltage was $3 \mathrm{~V}$ and the capillary temperature $195{ }^{\circ} \mathrm{C}$. Spectra were recorded in positive ion mode between $\mathrm{m} / \mathrm{z} 120$ and 1500 . The mass spectrometer was programmed to do a series of three scans: a full mass, a zoom scan of the most abundant ion in the first scan and MS-MS of the most abundant ion, using a collision energy of 30. For the analysis of anthocyanin fractions the same HPLC conditions already mentioned were used. The HPLC system was connected to the probe of the mass spectrometer via the UV cell outlet, using PEEK tubing.

\section{Results and discussion}

New HPLC conditions have been developed for the separation of strawberry anthocyanins. The use of a C18 column end capped with a hydrophilic (polar) reagent and $0.1 \%$ TFA as aqueous solvent effected a good chromatographic resolution of anthocyanins using a low flow rate $\left(0.5 \mathrm{ml} \mathrm{min}^{-1}\right)$, compatible with the requirements of the MS detector. Figure 2 shows the HPLC chromatogram recorded at $520 \mathrm{~nm}$ corresponding to the complete strawberry anthocyanin profile.

Peaks 2 and 6 were respectively assigned to cyanidin 3-glucoside and pelargonidin 3-glucoside based on their relative elution order and absorption spectra obtained with the diode array detector, compared to data in our library and collected from the literature $[2,4,5]$. Further support for this identification was obtained from their ESI-MS and MS-MS after LC-MS analysis (Table 1).

Pigment 7 showed a positive molecular ion $\left[\mathrm{M}^{+}\right]$at $\mathrm{m} / \mathrm{z}$ 579.0, which gave MS-MS fragments at $\mathrm{m} / \mathrm{z} 271.3$ (pelargonidin) and 432.9 (loss of a rhamnose residue). This fragmentation pattern was consistent with that indicated by Giusti et al. [13] for anthocyanin C3-rutinosides. In order to obtain additional support that allowed confirmation of its rutinoside nature, pigment 7 was isolated by HPLC and further submitted to sequential acid hydrolysis according to the procedure described by Giusti and Wrolstad [14]. So far as the degradation occurred, the original peak disappeared, emerging as the 3 -glucoside derivative and the aglycon peaks. No peaks that could be attributed to rhamnose derivatives were observed in any of the stages of the cleavage, thus allowing the ruling out of the theoretical possibility that the glucose and rhamnose residues were located at different po- 
Fig. 2 HPLC chromatogram $(520 \mathrm{~nm})$ showing the anthocyanin profile of a strawberry extract. See Table 1 for peak identification

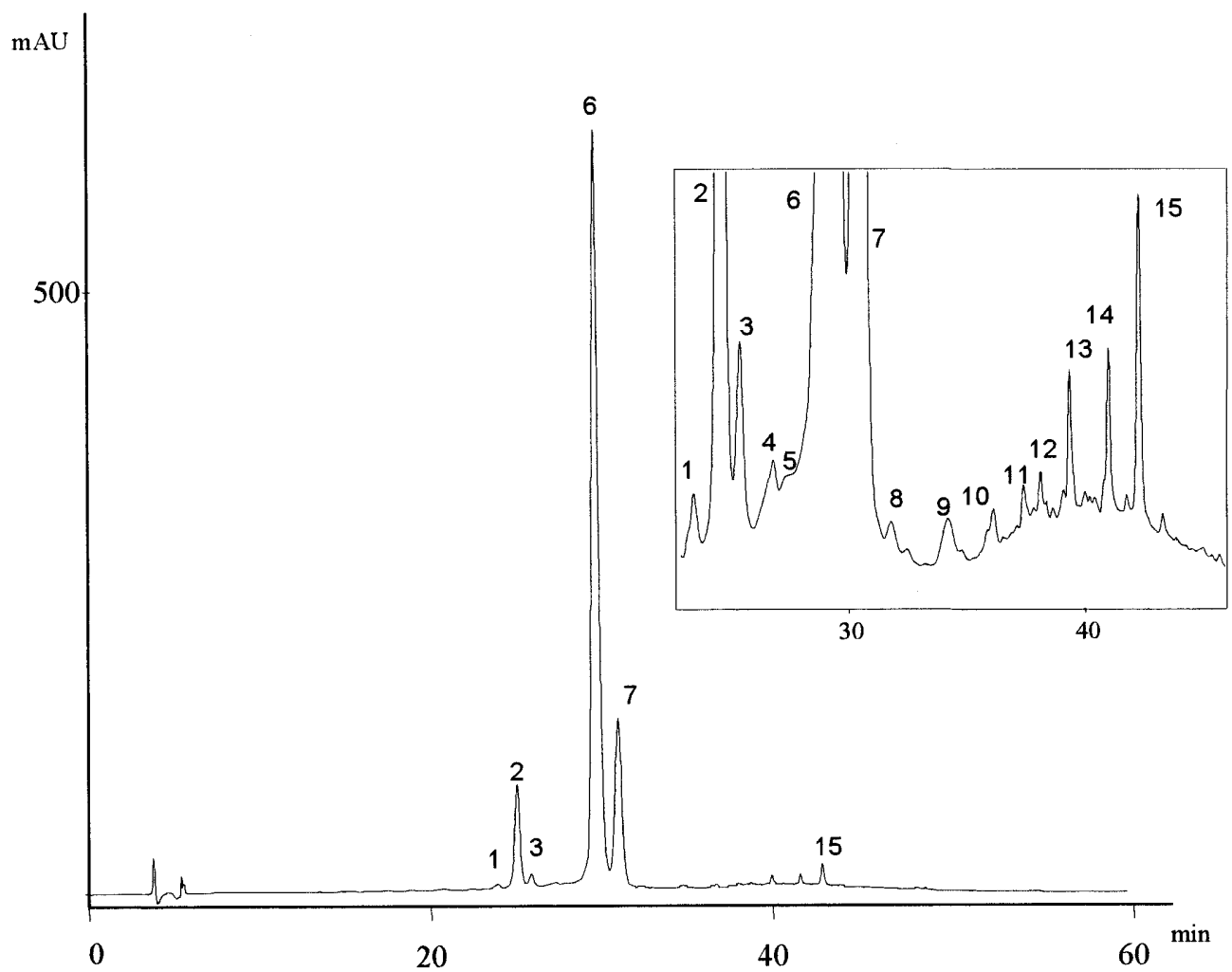

Table 1 Retention time $(R T)$, wavelengths of maximum absorption $\left(\lambda_{\max }\right)$, molecular ion, fragmentation pattern and tentative identification of the anthocyanins detected in the strawberry extract. $P g$ Pelargonidin, $C y$ cyanidin, $n . a$. not available

\begin{tabular}{|c|c|c|c|c|c|}
\hline Peak & $\mathrm{RT} \min$ & $\lambda_{\max } \mathrm{nm}$ & $\begin{array}{l}\text { Molecular ion } \\
{\left[\mathrm{M}^{+}\right] \mathrm{m} / \mathrm{z}}\end{array}$ & $\begin{array}{l}\mathrm{MS}^{2} \text { of } \\
{\left[\mathrm{M}^{+}\right] \mathrm{m} / z}\end{array}$ & Tentative identification \\
\hline 1 & 23.4 & $500-502$ & n.a. & & $\mathrm{Pg}$ derivative \\
\hline 2 & 24.6 & 515 & 449.0 & 287.1 & Cy 3-glucoside \\
\hline 3 & 25.4 & 515 & 595 & $448.6,287.2$ & Cy 3-rutinoside \\
\hline 4 & 26.8 & 500 & 595.2 & $432.9,271.1$ & Pg diglucoside \\
\hline 5 & 27.4 & 524 & 697.0 & $535.2 ; 449.1 ; 287.3$ & Cy 3-malonylglucose-5-glucose \\
\hline 6 & 29.1 & 502 & 433.1 & 271.2 & Pg 3-glucoside \\
\hline 7 & 30.5 & 503 & 579.0 & $432.9,271.3$ & Pg 3-rutinoside \\
\hline 8 & 31.8 & 502 & n.a. & & Pg derivative \\
\hline 9 & 34.2 & 503 & 549.0 & 271.2 & Pg 3-malylglucoside \\
\hline 10 & 36.1 & 518 & n.a. & & Cy derivative \\
\hline 11 & 37.4 & 503 & 607.2 & 271.2 & $\begin{array}{l}\text { Pg disaccharide (hexose+pentose) } \\
\text { acylated with acetic acid }\end{array}$ \\
\hline 12 & 38.1 & 503 & 607.1 & 271.3 & $\begin{array}{l}\text { Pg disaccharide (hexose+pentose) } \\
\text { acylated with acetic acid }\end{array}$ \\
\hline 13 & 39.4 & 504 & n.a. & & Pg derivative \\
\hline 14 & 41.0 & 504 & n.a. & & Pg derivative \\
\hline 15 & 42.3 & 504 & 475.0 & 271.2 & Pg 3-acetylglucoside \\
\hline
\end{tabular}

sitions in the anthocyanin structure (e.g. 3,5 or 3,7-substitutions). These observations confirmed it as pelargonidin 3-rutinoside. Its location in the chromatogram, immediately after pelargonidin 3-glucoside and the fact of its being a major anthocyanin in strawberry are in agreement with the observations made by other authors [2, 4, 5]. Pelargonidin-3-arabinoside, claimed to be the third major pigment in strawberry by Fiorini [9] and Goiffon et al. [10], was not detected by LC-MS when a search for its molecular ion $(\mathrm{m} / \mathrm{z} 403)$ was made either in the crude strawberry extract or in any of the fractions further obtained.

The UV-visible spectra of the remaining pigments obtained with the DAD detector showed that all of them were pelargonidin and cyanidin-based anthocyanins (Table 1). The shift observed in their $\lambda_{\max }$ in the visible region of the spectra of the compounds that elute at greater retention time is explained by the increase in the acetonitrile percentage in the mobile phase [6]. In order to obtain further information about these minority pigments, 

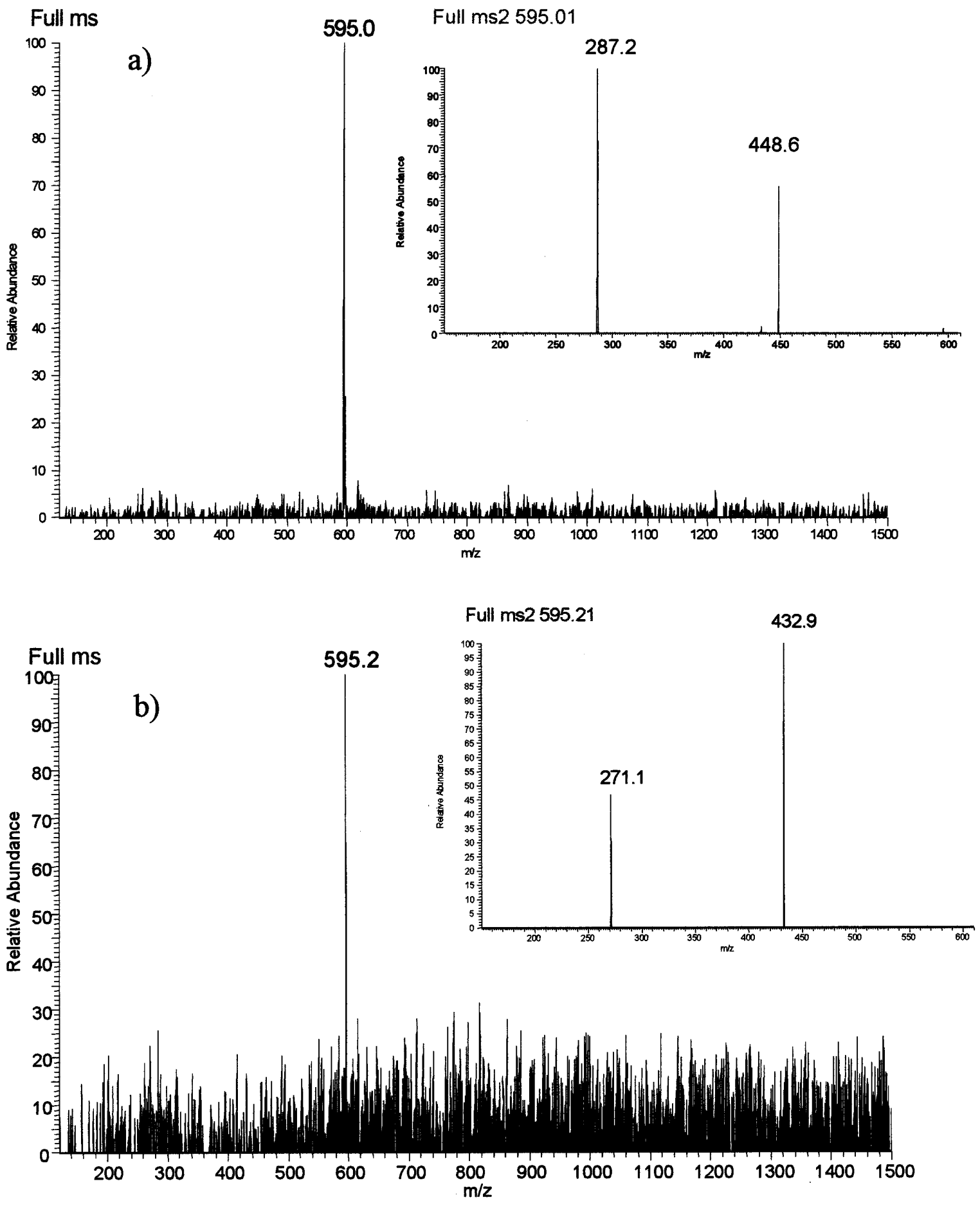

Fig. 3a, b Full mass spectrometry (MS) and MS/MS spectra obtained in positive ion mode for a peak 3, assigned to cyanidin 3rutinoside, and b peak 4 assigned to a pelargonidin diglucoside

the crude extract was fractionated by column chromatography. Three simpler and clear fractions were collected from which suitable MS spectra could be obtained. Pigments 3, 4, 5 and 7 (pelargonidin 3-rutinoside) were collected in fraction A (20-40\% ethanol); pigments 2 (cyanidin 3-glucoside), 9 and 15 eluted in fraction B (up to $80 \%$ ethanol), and pigments 11 and 12 in fraction $\mathrm{C}$ (up to $95 \%$ ethanol). The majority anthocyanin, pelargonidin 3-glucoside (peak 6), was present in all three fractions, although it reached its highest levels in the fraction $\mathrm{B}$. The remaining anthocyanin peaks $(1,8,10,13$ and 14) could not be obtained in any of the fractions in sufficient quantities.

Pigments 3 and 4 showed identical molecular ions at $\mathrm{m} / \mathrm{z}$ 595, but their fragmentation patterns were different, showing that they derived from cyanidin and pelargonidin respectively (Fig. 3), which was also confirmed from their UV-visible spectra (Table 1). Pigment 3 was as- 

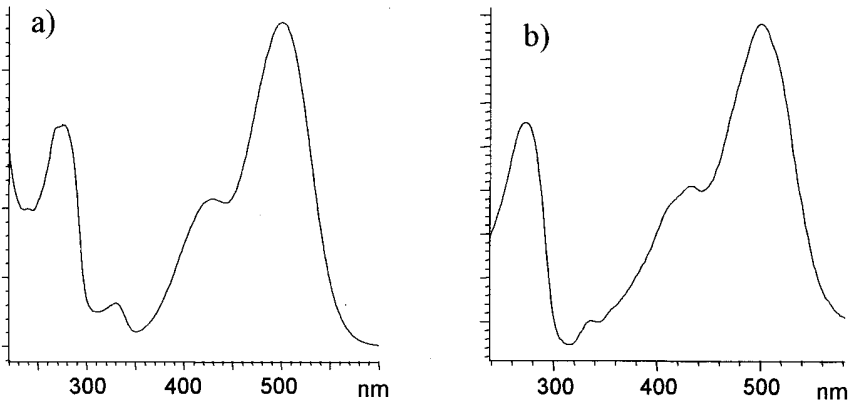

Fig. 4a, b UV-visible spectra of a peak 6 (pelargonidin 3-glucoside), and b peak 4 (pelargonidin diglucoside)

signed to cyanidin 3-rutinoside according to its fragmentation pattern (Fig. 3a), which was consistent with that indicated by Giusti et al. [13] for anthocyanin C3-rutinosides. This identity was also coherent with its location in the chromatogram and its proportions with regard to cyanidin 3-glucoside (peak 2), which follow a similar pattern to those observed for pelargonidin 3-rutinoside (peak 7) in relation to pelargonidin 3-glucoside (peak 6).

The MS-MS spectrum of pigment 4 (Fig 3b) allowed its identification as a pelargonidin derivative containing two hexose substituents. Each of the sugar residues must be located at different positions in the anthocyanidin structure, as only one peak corresponding to the aglycon should have been observed after MS-MS fragmentation if they had constituted a disaccharide [13]. The greater polarity of the compound with regard to pelargonidin 3glucoside also supports its diglycoside nature, as well as some differences observed in its UV-visible spectrum when compared to those of the pelargonidin C3-substituted derivatives (Fig. 4). One is the lack of the flattopped double peak at $270-280 \mathrm{~nm}$, and the other the existence of a shoulder instead of a defined peak at $330 \mathrm{~nm}$. According to Bakker et al. [4] both characteristics would be typical of a diglucoside. Thus, the compound could be pelargonidin 3,5- or 3,7-diglucoside, although the possibility of one or two of the hexose residues being different to glucose cannot be discarded either. A pelargonidin-based anthocyanin with similar RPHPLC retention characteristics was also detected by Bakker et al. [4], but no indication about its sugar substitution pattern was made.

The UV-visible spectrum of pigment 9 was similar to that of pelargonidin 3-glucoside but showed increased absorbance at $280 \mathrm{~nm}$. Its MS spectrum revealed a molecular ion at $\mathrm{m} / \mathrm{z}$ 549, which would match the structure of pelargonidin 3-glucoside acylated with malic acid. An identical molecular mass of 549 was obtained by Bakker et al. [4] for an anthocyanin separated from strawberries. In combination with its UV-visible spectral characteristics, those authors tentatively identified the compound as cyanidin 3-glucoside succinate. Such an identification must be discarded in our case, since the mass and absorption spectra of the pigment clearly assigned it as a pelargonidin derivative. Acylated C3-substituted anthocyanins are reported to produce only one fragment corresponding to the aglycon in their fragmentation [13], as observed in the MS-MS spectrum of peak 9, and they also have longer retention times than their respective non-acylated derivatives. All these characteristics al-

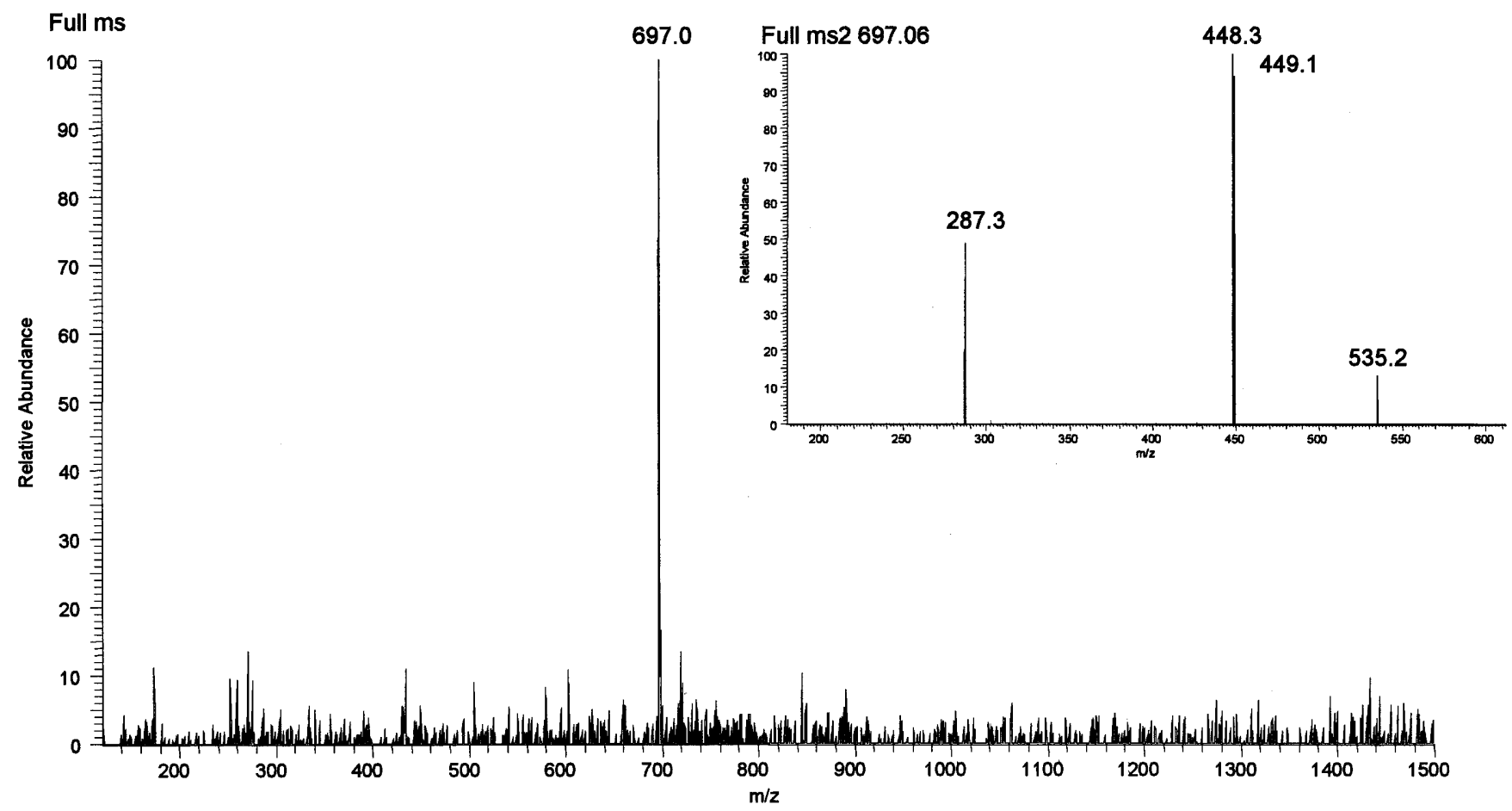

Fig. 5 Full MS and MS/MS spectra obtained in positive ion mode for peak 5 tentatively identified as a cyanidin malonyldiglucoside 
lowed us to assign this pigment to pelargonidin 3-malylglucoside. The presence of the malyl residue would also explain the increased absorbance observed at $280 \mathrm{~nm}$ compared to that of pelargonidin 3-glucoside. The existence of such an anthocyanin in nature is reported by Mazza and Miniati [11], but according to our knowledge it is here reported in strawberry for the first time. Similar arguments as for pigment 9 can be used for the identification of pigment 15, whose elution characteristics and absorption and mass spectra (Table 1) are coherent with pelargonidin 3-acetylglucoside. Such a compound had already been tentatively identified in strawberries by Hong and Wrolstad [2].

Pigments 11 and 12 presented identical molecular ions (Table 1). The production in their MS-MS spectra of only one fragment corresponding to the aglycon, indicated that they were also $\mathrm{C} 3$-substituted anthocyanins. A possibility that would match their molecular mass is the substitution of pelargonidin by a hexose+pentose disaccharide acylated with acetic acid. The detection of two compounds may be explained by the presence of different sugar substituents or, more probably, by the existence of a different linkage between the pentose and hexose residues in each of the pigments. Pelargonidin 3-sambubioside (i.e., $O$ - $\alpha$-L-xylosyl-( $1 \rightarrow 2$ )-glucoside), 3-glucosylxyloside and 3-xylosylglucoside have been described in different fruits [11, 15, 16, 17], but none of them has been cited in strawberries nor have their forms acylated with acetic acid been described.

Peak 5 appeared as a shoulder in the chromatogram of the strawberry extract (Fig 2); however, it could be obtained as a defined peak in fraction A with sufficient quality for obtaining its absorption and mass spectra, which clearly assigned it as a cyanidin derivative (Table 1). The molecular ion obtained matches a cyanidin dihexoside derivative acylated with malonic acid, which is also in agreement with the fragment ions observed in its MS-MS spectrum (Fig. 5) that would correspond to cyanidin malonylhexoside $(\mathrm{m} / \mathrm{z}$ at 535.2$)$, cyanidin hexoside $(\mathrm{m} / \mathrm{z}$ at 449.1$)$ and cyanidin $(\mathrm{m} / \mathrm{z}$ at 287.3$)$. According to this fragmentation pattern the hexose residues should be located at different positions in the anthocyanidin structure and thus it could be tentatively assigned to cyanidin 3-malonylglucose-5-glucose. Should this identity be confirmed, cyanidin 3,5-diglucoside could also have been expected to occur in strawberry. Such a diglucoside should elute at shorter retention time than cyanidin 3-glucoside and, therefore, it may correspond to any of the very small peaks that are observed in the base- line around 20-23 min in the chromatogram of Fig. 2, about which no information was obtained. The presence of a malonyl residue would decrease the polarity of cyanidin 3,5-diglucoside, which would be consistent with the location of peak 5 in the chromatogram. No references to a cyanidin malonyldiglucose have been found, either in strawberries or other plants.

The quality of the remaining peaks marked in the chromatogram of Fig 2 was only sufficient to assign them as pelargonidin derivatives $\left(\lambda_{\max }\right.$ at $\left.500-504 \mathrm{~nm}\right)$ in the cases of peaks $1,8,13$ and 14 , and as a cyanidin derivative for peak $10\left(\lambda_{\max }\right.$ at $\left.518 \mathrm{~nm}\right)$, but no further information could be obtained that allowed speculation about their structure.

Acknowledgements Thanks are due to Junta de Castilla y León (Spain) for financial support (project reference SA086/01). Author Lopes da Silva is funded by the European Commission and Government of Portugal through the Programme PRODEP III.

\section{References}

1. Timberlake C, Bridle P (1982) Distribution of anthocyanins in food plants. In: Markakis P (ed) Anthocyanins as food colors. Academic, New York, pp 126-162

2. Hong V, Wrolstad R (1990) J Agric Food Chem 38:708-715

3. Bakker J, Bridle P, Koopman A (1992) J Agric Food Chem 60:471-476

4. Bakker J, Bridle P, Bellworthy S (1994) J Sci Food Agric 64:31-37

5. Bridle P, García-Viguera C (1997) Food Chem 59:299-307

6. Hebrero E, Santos-Buelga C, Rivas-Gonzalo J (1988) Am J Enol Vitic 39:227-233

7. Hebrero E, García-Rodríguez C, Santos-Buelga C, RivasGonzalo J (1989) Am J Enol Vitic 40:283-291

8. Hong V, Wrolstad R (1990) J Agric Food Chem 38:698-708

9. Fiorini M (1995) J Chromatogr A 692:213-219

10. Goiffon J-P, Mouly P, Gaydou E (1999) Anal Chim Acta 382:39-50

11. Mazza G, Miniati E (1993) Anthocyanins in fruits, vegetables and grains. CRC, Boca Raton, USA, pp 108-109

12. Glaßgen W, Wray V, Strack D, Metzger J, Seitz H (1992) Phytochemistry 31:1593-1601

13. Giusti M, Rodríguez-Saona L, Griffin D, Wrolstad R (1999) J Agric Food Chem 47:4657-4664

14. Giusti M, Wrolstad R (1996) J Food Sci 61:322-326

15. Strack D, Wray V (1994) The anthocyanins. In: Harborne J (ed) The flavonoids - advances in research since 1986. Chapman, London, pp 1-22

16. Hrazdina G (1982). Anthocyanins. In: Harborne J, Mabry T (eds) The flavonoids - advances in research. Chapman, London, pp 135-188

17. Harborne J, Grayer R (1988) The Anthocyanins. In: Harborne J (ed) The flavonoids - advances in research since 1980 . Chapman, London, pp 1-20 